\title{
Caracterizar epidemiologicamente e referir os diagnósticos de enfermagem ao paciente acometido por leishmaniose visceral em Santarém-PA
}

To characterize epidemiologically and refer nursing diagnoses to patients affected by visceral

\author{
leishmaniasis in Santarém-PA
}

Caracterizar epidemiológicamente y referir el diagnóstico de enfermería a pacientes afectados por leishmaniasis visceral en Santarém-PA

Recebido: 03/12/2021 | Revisado: 09/12/2021 | Aceito: 10/12/2021 | Publicado: 17/12/2021

Claudia Maria Pereira dos Santos
ORCID: https://orcid.org/0000-0003-2557-4775
Centro Universitário da Amazônia, Brasil
E-mail: claudiapareirapds@ gmail.com
Claudineia Alves de Souza
ORCID: https://orcid.org/0000-0003-4590-2238
Centro Universitário da Amazônia, Brasil
E-mail: claudia.alves.stm@ @mail.com
Renilson Venâncio Barbosa
ORCID: https://orcid.org/0000-0003-4133-7147
Centro Universitário da Amazônia, Brasil
E-mail: renilsonelvis@ gmail.com
Maria Conceição Cavalcante Farias
ORCID: https://orcid.org/0000-0002-2519-1814
Centro Universitário da Amazônia, Brasil
E-mail: concefarias@ yahoo.com.br

\section{Resumo}

Introdução: A leishmaniose visceral é uma doença zooantroponose endêmica, causada por um protozoário climorfico trypapanossomatidae e sua transmissão para humanos ocorre através de vetores artropoles dos gêneros lutzonia phebotomia. Metodologia: Trata-se de um estudo qualitativo, transversal, retrospectivo documental e descritivo realizado através de coleta de dados no sistema de informação de notificação de doenças de notificação compulsória TABNET - Datasus e para elaboração dos diagnósticos de enfermagem foi usado NANDA-I 2018-2020. Resultado e Discussão: Os resultados mostram que no período de 2010 a 2019 foram notificados e confirmados 75 casos de leishmaniose visceral no município de Santarém Pará com maior incidência entre os anos de 2010 a 2013 com $44 \%$ dos casos, o sexo masculino apresentou taxa de $68 \%$ dos casos, a faixa etária entre 0-9 anos apresentaram 49\% dos casos e a zona urbana estão pessoas entre os mais infectados com $60 \%$ dos casos, os diagnósticos de enfermagem que mais comuns são nutrição desequilibrada: menor do que as necessidades corporais, conhecimento deficiente, risco de desequilíbrio na temperatura corporal. Conclusão: O estudo em questão identificou que a população do município de Santarém-PA apresentou maior número dos casos no sexo masculino e os casos com maior incidência ocorreu na zona urbana devido à infraestrutura inadequada que acaba contribuindo para infecção da leishmaniose visceral humana.

Palavras-chave: Leishmaniose visceral; Epidemiologia; Diagnósticos de Enfermagem.

\begin{abstract}
Introduction: Visceral leishmaniasis is an endemic zooanthroponosis disease, caused by a climorphic protozoan trypapanossomatidae and its transmission to humans occurs through arthropole vectors of the genus lutzonia phebotomy. Methodology: This is a qualitative, cross-sectional, retrospective documentary and descriptive study carried out through data collection in the TABNET compulsory notification information system - Datasus and NANDA-I 2018- was used to prepare nursing diagnoses. 2020. Results and Discussion: The results show that in the period from 2010 to 2019, 75 cases of visceral leishmaniasis were notified and confirmed in the municipality of Santarém Pará with a higher incidence between the years 2010 to 2013 with $44 \%$ of cases, males had a rate of $68 \%$ of cases, the age group between 0-9 years presented $49 \%$ of cases and the urban area are people among the most infected with $60 \%$ of cases, the most common nursing diagnoses are unbalanced nutrition: less than the needs body, deficient knowledge, risk of imbalance in body temperature. Conclusion: The study in question identified that the population of the municipality of Santarém-PA had a higher number of cases in males and the cases with higher incidence occurred in urban areas due to inadequate infrastructure that ends up contributing to the infection of human visceral leishmaniasis.
\end{abstract}

Keywords: Visceral leishmaniasis; Epidemiology; Nursing Diagnoses. 


\section{Resumen}

Introducción: La leishmaniasis visceral es una enfermedad de zooantroponosis endémica, causada por un protozoario climórfico tripapanossomatidae y su transmisión al ser humano se produce a través de artrópolos vectores del género lutzonia febotomía. Metodología: Se trata de un estudio cualitativo, transversal, retrospectivo, documental y descriptivo realizado mediante recolección de datos en el TABNET - Sistema de información de notificación obligatoria Datasus y NANDA-I 2018- se utilizó para la elaboración de diagnósticos de enfermería.2020. Resultados y Discusión: Los resultados muestran que en el período de 2010 a 2019, se notificaron y confirmaron 75 casos de leishmaniasis visceral en la ciudad de Santarém Pará con una mayor incidencia entre los años 2010 a 2013 con $44 \%$ de los casos, los varones presentaron una tasa del 68\% de los casos, el grupo de edad entre 0-9 años presentó el 49\% de los casos y el área urbana son las personas entre las más infectadas con el 60\% de los casos, los diagnósticos de enfermería más comunes son la nutrición desequilibrada: menos de las necesidades corporales, conocimiento deficiente, riesgo de desequilibrio en la temperatura corporal. Conclusión: El estudio en cuestión identificó que la población del municipio de Santarém-PA tenía un mayor número de casos en varones y los casos con mayor incidencia ocurrieron en áreas urbanas debido a una infraestructura inadecuada que termina contribuyendo a la infección de leishmaniasis visceral humana.

Palabras clave: Leishmaniasis visceral; Epidemiología; Diagnósticos de enfermería.

\section{Introdução}

A leishmaniose visceral é classificada como uma Zooantroponose endêmica as regiões brasileiras onde os fatores para a infecções estão o desmatamento nas zonas rurais e urbanização dos vetores devido a ocupação desordenada dos espaços urbanos (Aguiar, 2017).

É uma patologia de forma crônica, em seu estado grave pode levar a óbito, quando não é feito um tratamento adequado. A leishmaniose é considerada um problema de saúde pública, representando dificuldade para a equipe de saúde, dados epidemiológicos e um fator importante para o planejamento efetivo de estratégicas no controle (Castro, 2016).

É uma enfermidade provocada por um protozoário Climorfico trypapanossomatidae do complexo Leishmania donavoni, que na forma amastigota, o parasita afeta o sistema fagocitico- mononuclear contaminando mamíferos incluindo o homem. Sua transmissão é através de vetores artropoles dos gêneros Lutzomia e Phlebotomeim pela picada do mosquito no homem. A LV também é conhecida popularmente como calazar, o cachorro é o seu principal reservatório doméstico do protozoário, junto com roedores domésticos e canídeos silvestres (Slivinski, 2019).

O protozoário que provoca a leishmaniose é capaz de se assegurar em um estado de autocontrole com o sistema imunológico dos indivíduos hospedeiro, sendo conhecido como cura clínica, quando aparece a doença nesses indivíduos quando estão em condição de imunossupressão o parasita se torna oportunista, e a quimioterapia e a primeira abordagem para conduzir a doença, a quimioterapia não se aplica a indivíduos assintomáticos (Souza, 2020).

Para Lewgoy, 2019 o Brasil lidera o ranque dos países das Américas com maior numero de casos de LV, no Brasil ocorreu a primeira notificação desta infecção em 1917 e foi colocada como uma doença endêmica rural por muito tempo, em 1980 ocorreu urbanização da doença no país, as políticas de eliminação dos vetores especificamente de insetos estão entre os meios de controle de epidemias.

Segundo dados do TABNET - Datasus, na última década o brasil notificou 36.409 casos, estando em primeiro lugar a região nordeste com maior numero de casos com 19.809, seguido da região sudeste com 6.951, logo depois a região norte com 6.599 e a região centro oeste com 2.949, a região com menor numero durante esse período foi a região sul com 101 casos. O Pará nesse mesmo período foram notificados 3.465 casos, as faixas etárias mais afetadas foram de 1 a 4 anos de idade com $31,8 \%$ e as pessoas com idades $>65$ anos apresentam menor numero de casos.

A domesticação de animais contribui para o acréscimo de notificações de casos na comunidade, o sacrifício de cães como indicação para diminuir o contágio de LVH, se iniciou na Palestina com amparo de estados centralizados, no entanto alguns países discordaram do sacrifício de animais que moram na rua, como estratégia de saúde. (Silva, 2020) 
A leishmaniose visceral é diagnosticada através de exames clínicos e laboratoriais, sendo o clínico deve ser associado com os sintomas complexos são necessários exames laboratoriais para confirmar o diagnóstico parasitológico, envolve a punção de órgãos que a presença do parasita, torna-se um procedimento invasivo pelo fato de retirar a pele lesionado ou parte dela. No teste molecular e realizado a retirada do DNA do agente etiológico para aplicação da técnica de reação em cadeia de polimerase (PCR). Os testes imuno-histoquimico é um método que precisa de uma parte do tecido para colocar na lâmina histológica junto com anticorpos específicos para se Liga e ao parasito por uma ligação antígeno-anticorpo. O sorológico é feito no método ELISA, usando o soro do hospedeiro para observação da formação do anticorpo (Cavalcante, 2020).

O tratamento da LV é considerado muito complexo, sendo obrigatório um acompanhamento ambulatório no período em que o indivíduo estiver fazendo uso de medicamento, as vias de administração são vias intramuscular e intravenosa. Os medicamentos usados são os antimoniatos pentavalentes, como anfotericina B é um medicamento microbiano sistêmico usado em via intravenosa e pode se associado com o deaxicolato de sódio, nos protozoários do Reino leishmania o AmB atua diretamente na membrana do parasita (Ribeiro, 2021).

O processo de enfermagem e uma ferramenta para tornar a assistência organizada com o objetivo de ajudar os enfermeiros no âmbito que envolve a promoção e qualidade de cuidados prestados aos pacientes, o cuidado de enfermagem deve ser baseado em evidencias usando o raciocínio clinico e elaborar diagnósticos de enfermagem e avaliar os resultados das intervenções. Wanda de Aguiar Horta por volta dos anos 80 incluiu para fins didáticos, se divide em etapas que são: Histórico de enfermagem (anamnese), Diagnostico de Enfermagem, Planejamento de Enfermagem, Implementação e Avaliação de Enfermagem, que dispõe da resolução COFEN n 358/2009 (Santos, et al 2017).

O papel do enfermeiro consiste em programas de prevenção nas comunidades para diminuir o numero de infectados usando metodologias de educação e prevenção, desenvolver atividades, realizar palestras educativas, auxiliar no planejamento, monitoramento das ações em saúde direcionando as intervenções para minimizar as complicações, avaliar sinais característicos da infecção e orientar sobre as medidas preventivas, atuando frente às estratégias de prevenção e elaboração de diagnósticos de enfermagem para prestar uma assistência com foco nas necessidades humanas básicas dos pacientes de forma individualizada (Santos, et al 2019).

O objetivo deste trabalho foi caracterizar quanto o perfil epidemiológico e sociodemográfico, pontando indicadores como: qualitativo de pessoas, faixa etária, sexo, zona de urbanização e referir os diagnósticos de enfermagem elaborados ao paciente acometido pela doença em questão.

\section{Metodologia}

Trata-se de uma pesquisa bibliográfica, qualitativo, documental, Praça, 2015, por meios de artigos publicados em sites seguros, Sistema de Informações de doenças de notificação compulsória TABNET - Datasus, para analise dos dados sobre o perfil epidemiológico no município de Santarém-Pará entre 2010 a 2019 e elaboração dos diagnósticos de Enfermagem segundo os sintomas baseado na NANDA-I definições e classificação 2018-2020.

O estudo em questão usou dados dos pacientes acometido por leishmaniose visceral em Santarém-Pa. Utilizaram-se como critério de inclusão usado casos que foram acometidos e notificados em Santarém Pará, de ambos os sexos, zona de residência, faixa etária e casos confirmados por ano e como critério de exclusão foram excluídos os dados de pacientes que foram infectados em outros municípios e estados do Brasil, fizeram parte do estudo dados de 75 casos. 


\section{Resultado e Discussão}

Os resultados mostram que foram confirmados 75 casos no município de Santarém Pará no período de 2010 a 2019 , os dados mostram que entre 2010 a 2012 teve o maior índice de infectados com 33 casos (44\%), 2013 a 2016 com 23 casos $(30,7 \%)$ e entre 2017 a 2019 com 19 casos $(25,3 \%)$, como mostra na Tabela 1.

Tabela 1- Ocorrência de pessoas acometidas por leishmaniose visceral entre 2010 a 2019 em Santarém-PA.

\begin{tabular}{ll}
\multicolumn{1}{c}{ Casos confirmados por Ano } & $\mathbf{N}^{\circ}(\%)$ \\
\hline $2010-2012$ & $33(44 \%)$ \\
$23(30,7 \%)$ \\
$2013-2016$ & $19(25,3 \%)$ \\
\hline \multicolumn{1}{c|}{ Casos confirmados por sexo } & $51(68 \%)$ \\
\hline Masculino & $24(32 \%)$ \\
\hline Feminino & \\
\hline \multicolumn{1}{c|}{ Casos Confirmados por faixa etária } & $37(49 \%)$ \\
\hline $10-19$ anos & $05(6,7 \%)$ \\
\hline $20-49$ anos & $31(41,3 \%)$ \\
\hline $60-79$ anos & $02(2,7 \%)$ \\
\hline Zona Rural & $30(40 \%)$ \\
\hline
\end{tabular}

Fonte: TABNET - Datasus.

Podemos observar que os dados encontrados neste estudo estão relacionados com Souza (2018), em um estudo realizado em Sobral-CE entre os anos de 2011 a 2015 onde mostra os casos em ordem decrescente durante os anos, de 2011 a 2013 foi o período com alto índice de casos com 73,8 \% do total e de 2014 a 2015 com menor índice com $26 \%$ do total.

Depois da tabulação de dados relacionada ao sexo dos pacientes em estudo, foi possível observar que $68 \%$ dos casos infectados foram do sexo masculino e $32 \%$ foram do sexo feminino como mostra na Tabela 1. A população mais afetada por leishmaniose visceral em Santarém Pará são as pessoas que residem em área urbana com 45 (60\%) dos casos e zona rural com $30(40 \%)$ dos casos como mostra na Tabela 1.

Quando se fala no texto acima que as pessoas do sexo masculino são acometidas com maior frequência condiz com o estudo de Souza (2018) realizado em sobral onde a população do sexo masculino apresentaram $68 \%$ dos casos, sendo que a patologia pode acometer ambos os sexos, porém, devido o homem está mais exposto aos vetores flebotominios são os mais acometidos.

Os dados acima citados corroboram com Menezes (2016), quando relata que os fatores de risco estão relacionados à característica socioeconômica e demográfica, com animais domésticos, insetos hematofagos, roedores, lote baldio, áreas verdes nas proximidades, coleta de lixo irregular, quintal com plantações e com Toledo (2017) em um estudo realizado em Araguaína 
entre 2007 a 2012, mostrou a maior incidência dos casos na área urbana são em periferias, onde a infraestrutura e saneamento básico são precários, nas áreas centrais e intermediárias da cidade ocorre um número menor de infecções.

A Tabela 1 apresenta o resultado de pessoas infectadas por leishmaniose visceral por faixa etária, onde as pessoas mais afetadas são crianças com idade < 9 anos contabilizando 33 casos (49\%), seguindo pela faixa etária de 20 - 59 anos com 31 casos $(41 \%)$ e depois de 10 - 19 anos com 05 casos $(6,7 \%)$ e por fim $60-79$ com 2 casos $(2,7 \%)$.

Os dados deste estudo relacionado a faixa etária se aproximam com Farias (2019), que relata em um estudo que o maior número de casos notificados são na faixa etária estão entre 0 a 14 anos, seguido por 20 e 49 anos.

\section{Diagnósticos de Enfermagem}

Os diagnósticos de enfermagem são usados na prática clínica de enfermagem em 1973, Em 1982 passou a ser denominar-se NANDA- Nort American Nursing Diagnosis association, expandindo pelos países com o objetivo de padronizar de modo internacional os diagnósticos de Enfermagem, para proporcionar uma estrutura para a organização de sua ciência, e preciso ter conhecimento e responsabilidade para elaborar os diagnostico de enfermagem à um paciente, pois eles estão relacionados a assistência de enfermagem individualizada, sendo o segundo processo de enfermagem e sendo privativo do profissional enfermeiro (Conceição, 2017).

A presença clínica pode ser na forma assintomática que ocorre quando o indivíduo afetado não apresenta manifestação de sintomas e na forma sintomática com quadro de febre, anemia, tosse, hepatoesplenomegalia, leucopenia, hipoalbuminemia, pancitopenia e hipergamaglobulinemia. Em casos mais grave da patologia o individuo presenta diarreia, emagrecimento, icterícia, vômito e edema periférico (Souza, 2018).

Tabela 2. Diagnósticos de Enfermagem segundo a NANDA-I 2018 - 2020.

\begin{tabular}{l|c|c}
\hline Diagnóstico de Enfermagem & Domínio & Classe \\
Hipertermia & 11 & 6 \\
$\begin{array}{l}\text { Nutrição desequilibrada menor que as } \\
\text { necessidades corporais }\end{array}$ & 2 & 4 \\
Risco de Função Hepática & 2 & 4 \\
Prejudicada & 5 & 4 \\
\hline \multicolumn{1}{c|}{ Conhecimento deficiente } & & \\
\hline
\end{tabular}

Fonte: Autores.

\section{Considerações Finais}

A leishmaniose visceral é uma infecção provocada por parasitas do gênero leishmania pode ser considerada fatal quando não é feito o tratamento correto, tendo como seu hospedeiro animal doméstico é transmitido pela picada do mosquito phlebotominae que são insetos que se alimentam de sangue, esse tipo de leishmaniose compromete os órgãos internos do individuo.

Entre os diagnósticos de Enfermagem foram elaborados baseados nos sintomas comuns tais como febre devido a infecção por leishmania sp que acaba aumentando a temperatura corporal uma resposta do sistema imunológico tentando ativar suas defesas e o emagrecimento devido a inapetência devido a alimentação insuficiente para a necessidade corporal. 
A queda dos casos nos últimos anos está diretamente ligada à competência dos profissionais de saúde que estão cada vez mais capacitados interromper a transmissão de forma precoce a doença cabe principalmente aos enfermeiros de unidades básicas de saúde, que atuam na promoção, prevenção e reabilitação dos pacientes.

Com esse estudo foi possível caracterizar o perfil dos indivíduos acometidos por leishmaniose visceral e zona de casos confirmados, crianças com idade $<5$ anos e entre 20 a 49 estão entre a faixa etária que são mais acometidas e do sexo masculino, contudo a população que residem em áreas urbanas tem maiores chances de ser contaminados por leishmaniose, por esse motivo e importante que essas pessoas que estão em zona de risco que apresentarem alguns sintomas procurem ajuda.

\section{Referências}

Aguiar, P. F \& Rodrigues, R. K.(2017). Leishmaniose visceral no brasil: artigo de revisão. Revista Unimontes cientifica, montes claros, v.19,n.1- jan/jun 2017. (ISSN 2236-5257). http://Rev.unimontes.br/index.php/unicienfica/artide/view/526

Alves, W. A \& Fonseca, D. S. Leishmaniose visceral humana: Estudo do perfil clínico-epidemiológico na região leste de Minas Gerais, Brasil. J. Health Biol sci. 2018; 6(2): 133-139.MG. DOI:10.1262/2317-3076jhbs.v6i2.1764.p133139.2018

Brasil, Ministério da saúde. Banco de dados do Sistema Único de Saúde-DATASUS. http://www.datasus.gov.br

Castro, J. M. et al. (2016). Conhecimento percepções de indivíduos em relação A leishmaniose visceral humana como novas ferramentas de controle. Ensaios cienc. Cien biol. Agrar. Saúde, v.20,n2,P.93-103,2016. http://www.redalyc.org/artículo.oa?id=26046651006

Cavalcante, M. V. T. et al. (2018) Leishmaniose visceral: métodos de diagnóstico laboratorial 2.00.00.00-6 - Ciências Biológicas 2.13.00.00-3 Parasitologia. https://eventos.set.edu.br/al_sempesq/article/view/10853/0\#: :text=H\%C3\%A1\%20 uma\%20suspeita\%20da\%20doen\%C3\%A7a,atrav\%C3\%A9s\%20da\%20intradermorrea\%C3\%A7\%C3\%A3o\%20de\%20Montenegro

Conceição, V. M. et al. Sistematizacao da assistência da Enfermagem: uma revisão integrativa. Revista Eletrônica Gestao saúde ISSN: $1982-4785$ (Brasilia) vol.08,n.03.set.2017.395-413 https://periodicos.unb.br/index.php/rgs/article/download/10310/9103/18510

Diagnostico de enfermagem da NANDA : definições e classificações 2018-2020/NANDA internacional. 11edição ed: Artmed-porto Alegre 2018.

Lewgoy, B. Mastraneelo, A. Beck, L. tonato político e biossegurança: dois regimes de governo da vida para a leishmaniose visceral canina no Brasil. P.145176. 2019 https://journals.openedition.org/horizontes/4423

Menezes, J. A. et al. Fatores de risco peridomicilares e conhecimento sobre leishmaniose visceral da população de formiga, Minas Gerais. SP. 2016 https://doi.org/10.1590/1980-5497201600020013

Ribeiro, L. P. F. et al. Tratamento empírico com desoxicolato de anfototerina B, em caso suspeito de leishmaniose visceral. Um relato de caso. Brasilian journal of development ISSN: 2525-8761.Belo Horizonte, MG. 2021 https://doi.org/10.34117/bjdv7n2-125

Praça, F. S. G. 08, n⿳ 1, p. 72-87, JAN-JUL, 2015. Revista Eletrônica "Diálogos Acadêmicos" (ISSN: 0486-6266) http://www.uniesp.edu.br/sites/_biblioteca/revistas/20170627112856.pdf

Santos, M. A. P. et al. Processo de enfermagem: Sistematização da assistência de enfermagem - SAE. Revista saúde em foco - edição nº - 2017 https://www.google.com/erl?sa=t\&source=web\&rct=j\&url=https://portal.unisepe.com.b

r/unifia/wpcontent/uploads/sites/10001/2018/06/075processodeemfermagem.pdf\&ved=2ahUKEwjq6omas0AhU0ppUCHXfhBiQQFnoECEEQAQ\&usg=AOv VAW1OPbxhFKuKglWT7xT1V5X

Santos, E. S. M. et al. Aspectos epidemiológicos da leishmaniose visceral. Revista eletrônica acervo saúde. ISSN 217-2091.vol.sup.25 e 959 https://doi.org/10.25248/reas.e959.2019

Silva, S. T. P. et al. Leishmaniose visceral humana: reflexões éticos e jurídicos acerca do controle do reservatório canino no Brasil. Rev. Bioética y Derecho no.39 Barcelona 2017 epub 02-nov- 2020. Lesion on-line ISSN 1886-5887 http://scielo.isciii.es/scielo.php?script=sci_arttext\&pid=S188658872017000100009

Souza, A. et al. Perfil epidemiológico dos casos de leishmaniose visceral em sobralCE de 2011 a 2015. SANARE, sobral-v.17,n.01,P.51-57,jan/jun. 2018 https://doi.org/10.36925/sanare.v17i1.1222

Souza, E. C. et al. Clinical presentation of visceral leishmaniosis in patients with HIV: analysis of disiose-related factors. Braz.J.Hea. Rev, Curitiba, v.3,n.2,P.1766-1777 mar/Apr. 2020. ISSN 2595-6825 https://doi:10.34119/bjhrv3n2-037

Slivinski, C. T. Analise crítica das ciências da saúde. Ponta Grossa, PR: Atena Editora; v3,2019 DOI10.22533/ah.et.782190710

Scopel, G. C \& Baronco, A. Focal Splenic lesions as an indicator of visceral leishmaniosis: A review. FAG. JOURNAL off Health 1 (2021) 72-78 https://doi.org/10.35984/fjh.v3i1.296

Toledo, C. R. S. et al. Vulnerabilidade a transmissão da leishmaniose visceral humana em áreas urbanas brasileira. Rev.Saude publica 51.15 maio 2017 https://doi.org/10.1590/S1518-87.2017051006532

Viegas, G. et al. Estudos de caso-perfil epidemiológico da leishmaniose visceral no município de João monlevade de 2015 a 2018 . Revista eumednet. Marzk 2019- ISSN-1696-8352 https://www.eumed.net/rev/oel/2019/03/leishmaniosevisceral.html 